\title{
Magnetohydrodynamic Flow of Viscous Fluid over a Non-Linearly Stretching Sheet
}

\author{
Yohannes Yirga*, Daniel Tesfay \\ Department Mathematics, Mekelle University, Mekelle, Ethiopia \\ Email: ${ }^{*}$ yohannesalbin@yahoo.com
}

Received 3 August 2014; revised 25 September 2014; accepted 27 October 2014

Copyright (C) 2014 by authors and OALib.

This work is licensed under the Creative Commons Attribution International License (CC BY). http://creativecommons.org/licenses/by/4.0/

(c) (i) Open Access

\begin{abstract}
In this paper, the Magnetohydrodynamic (MHD) Flow of Viscous Fluid over a Nonlinear Stretching Sheet is investigated numerically. The partial differential equations governing the flow are reduced to a non linear ordinary differential equations by using similarity transformations. The resulting transformed equations are numerically solved by an explicit finite difference scheme known as the Keller Box Method. The velocity profiles are determined and the effects of the magnetic parameter and non linear stretching parameter on the flow characteristics are investigated. In addition to this the numerical results for the local skin friction coefficients are computed. Comparison with the exact solution and previously reported analytic solutions is made and excellent agreement is noted. Moreover, the velocity profile obtained by Keller box method is in a better agreement to the exact solution than by the Homotopy Analysis Method. It is also found that, an increase in the magnetic parameter or non-linearity parameter causes a decrease in the velocity profile and velocity distribution.
\end{abstract}

\section{Keywords}

Magneto-Hydrodynamics, Non-Linear Stretching, Homotopy Analysis Method, Keller Box Method

Subject Areas: Fluid Mechanics, Partial Differential Equation

\section{Introduction}

The boundary layer flow of an incompressible fluids over a stretching sheet has many Engineering applications such as in manufacturing of sheeting materials through an extrusion process, aerodynamic extrusion of plastic sheets, the boundary layer along a liquid film in condensation process, hot rolling, paper production, glass-fiber production, metal spinning and drawing plastic films.

The boundary layer flow problems have been extensively studied numerically, analytically, theoretically as

${ }^{*}$ Corresponding author.

How to cite this paper: Yirga, Y. and Tesfay, D. (2014) Magnetohydrodynamic Flow of Viscous Fluid over a Non-Linearly Stretching Sheet. Open Access Library Journal, 1: e1030. http://dx.doi.org/10.4236/oalib.1101030 
well as experimentally. The theoretical study of magnetohydrodynamic (MHD) channel has been a subject of great interest due to its widespread applications in designing cooling systems with liquid metals, MHD generators, accelerators, pumps and flow meters. The solution for MHD flow through a flat channel is well known and is available in the text books of fluid dynamics. The boundary layer flow over a stretching sheet was first studied by Sakiadis [1]. Since then many investigators have extended the idea to different aspect of boundary layer flow problems. Accordingly, Crane [2] extended the study and obtained the analytic solution to the boundary layer equation of steady two dimensional flows due to a stretching sheet in a quiscent incompressible fluid. The flow inside a stretching channel or tube has been analyzed by Brady and Acrivos [3] and the flow outside the stretching tube by Wang [4]. Nadeem and Hussain [5] studied the MHD flow of a viscous fluid on a nonlinear porous shrinking sheet with homotopy analysis method. Sajid [6] investigated Homotopy analysis of stretching flows with partial slip. His result indicated that the velocity components decrease with an increase in the slip parameter for both planar and axisymmytric flows. Nadeem [7] studied the boundary layer flow of nanofluid over an exponentially stretching surface with the effects of suction/injection parameter. He observed that velocity field increases with the increase in the suction/injection parameter and the suction causes the reduction of the boundary layer. Sharidan [8] has presented similarity solution analysis to investigate the unsteady boundary layer flow over a stretching sheet for a special distribution of the stretching velocity and surface heat flux. Cortell [9] studied viscous flow and heat transfer over a nonlinearly stretching sheet. Alinejad and Samarbakhsh [10] studied viscous flow over nonlinearly stretching sheet with effects of viscous dissipation. Several studies on the steady and unsteady flows due to stretching sheet has been considered by Devi et al. [11], Anderssson et al. [12], Nazar et al. [13], Elbashbeshy and Bazid [14], and Ishak et al. [15], Wubshet et al. [16], Turkyilmazoglua and Popb [17] and Xua et al. [18].

Most problems in fluid mechanics are non linear. It is very important to develop an efficient method to solve them. Up to now, several analytic and numerical methods have been used. In tradition, the perturbation method is used to give analytic approximations of non linear problems. This technique uses asmall/large parameter assumption. Various non perturbation methods such as Lyapunov's artificial small parameter method [19], $\delta-$ expansion method [20], the Adomains method [21] and recently the homotopy analysis method developed by Liao [22] have been used to solve the flow problems.

The main objective of the present study is to find a numerical solution for the MHD boundary layer flow over a non-linear stretching sheet. By making use of computational software, Matlab an accurate numerical solution for the velocity profile is obtained using a powerful, easy to use method called the Keller box method. This method has already been successfully applied to several non linear problems corresponding to parabolic partial differential equations. As discussed in [23] the exact Discrete Calculus associated with the Keller-Box scheme is shown to be fundamentally different from all other mimic (physics capturing) numerical methods. The boxscheme of Keller is basically a mixed finite volume method, which consists in taking the average of a conservation law and of the associated constitutive law at the level of the same mesh cell.

The paper is outlined as follows, in Section 2 the problem is formulated and the similarity transformation is used to reduce the formulated partial differential equation in to a non linear ordinary differential equation and a Keller box method is used to solve the problem; in Section 3 the resulted solutions are discussed in detail both numerically and graphically and the solutions are compared with the analytic solution of the same problems done by Hayat [24] using the modified decomposition method and Pade approximation; and by Abdoul [25] using homotopy analysis method (HAM). Finally in Section 4, some concluding remarks are given.

\section{Mathematical Formulation}

Consider a steady two-dimensional MHD flow of viscous and incompressible fluid over a nonlinearly stretching sheet at $y=0$. The fluid is electrically conducting under the influence of an applied magnetic field $B(x)$ normal to the non-linear stretching sheet. The induced magnetic field is neglected. With these assumptions the governing equations for the flow problem are

$$
\begin{gathered}
\frac{\partial u}{\partial x}+\frac{\partial v}{\partial y}=0 \\
u \frac{\partial u}{\partial x}+v \frac{\partial u}{\partial y}=v \frac{\partial^{2} u}{\partial y^{2}}-\frac{\sigma B^{2}(x)}{\rho} u
\end{gathered}
$$


where $u$ and $v$ are velocity components in the $x$ and $y$ directions respectively. $v, \rho$ and $\sigma$ are the kinematic viscosity, density and electrical conductivity of the fluid respectively.

In Equation (2), the external electric field and the polarization effects are negligible and

$$
B(x)=B_{0} x^{\frac{n-1}{2}}
$$

The boundary conditions corresponding to the non-linear stretching sheet are given by

$$
u(x, 0)=c x^{n}, v(x, 0)=0, \quad u(x, y) \rightarrow 0 \text { as } y \rightarrow \infty
$$

Introducing the following similarity transformations

$$
\eta=\sqrt{\frac{c(n+1)}{2 v}} y x^{\frac{n-1}{2}}, u=c x^{n} f^{\prime}(\eta), v=-\sqrt{\frac{c v(n+1)}{2}} x^{\frac{n-1}{2}}\left[f(\eta)+\frac{n-1}{n+1} \eta f^{\prime}(\eta)\right]
$$

Making use of Equation (5), the continuity Equation (1) is automatically satisfied and the momentum Equation (2) and the boundary conditions (3) reduce to

$$
f^{\prime \prime \prime}+f f^{\prime \prime}-\beta f^{\prime 2}-M f^{\prime}=0
$$

With boundary conditions

$$
f(0)=0, f^{\prime}(0)=1, f^{\prime}(\infty)=0
$$

where $M$ is the magnetic parameter and $\beta$ is the non linearity parameter. They are given by

$$
M=\frac{2 \sigma B_{0}^{2}}{\rho c(n+1)}, \quad \beta=\frac{2 n}{n+1}
$$

\section{Numerical Solution}

The non linear boundary value problem represented by Equations (6) and (7) is solved numerically using a very efficient finite difference scheme known as Keller box method. The scheme employed is the box method developed by Keller [23]. This method has been shown to be particularly accurate for parabolic problems. It is much faster, easier to program and it is chosen because it seems to be the most flexible of the common methods, being easily adaptable to solving equations of any order. The Keller-box method is essentially an implicit finite difference scheme, which has been found to be very suitable in dealing with nonlinear problems. Details of the method may be found in many recent publications, and here we have used the procedure outlined in Cebeci and Pradshaw [26]. One of the basic ideas of the box method is to write the governing system of equations in the form of a first order system. First derivatives of $u$ and other quantities with respect to $\eta$ must therefore be introduced as new unknown functions. With the resulting first order equations, the "cantered-difference" derivatives and averages at the midpoints of net rectangles and net segments are used, as they are required to get accurate finite difference equations. The resulting finite difference equations are implicit and nonlinear. Newton's method is first introduced to linearize the nonlinear system of equations before a block-tridiagonal factorization scheme is employed on the coefficient matrix of the finite difference equations. The solution of the linearized system of difference equations can be obtained in a very efficient manner by using the block-elimination method [26]. In this study a uniform grid of size $\Delta \eta=0.01$ is chosen to satisfy the convergence criteria of $10^{-5}$, which gives about a four decimal places accuracy for most of the prescribed quantities.

\section{Results and Discussions}

The transformed non linear momentum Equation (6) subjected to the boundary condition (7) was solved numerically using Keller box method, which is described in Cebeci and Bradshaw [26]. The results of the velocity distributions for different values of the magnetic parameter $M$ and the non linear parameter $\beta$ are presented in graphs, while the values of the skin friction coefficients for some values of the parameters are given in a table.

Table 1 and Table 2 show the values of the skin friction coefficient $-f^{\prime \prime}(0)$ for some values of the parameters $M$ and $\beta$. The values of the skin friction coefficient $\$ f\{"\}(0) \$$ obtained in this study are compared with 
Table 1. Comparison of the skin friction coefficient $-f^{\prime \prime}(0)$ obtained by Keller Box method with HAM, ADM-Pade and Exact solution for some values of $M$, when $\beta=1$.

\begin{tabular}{ccccc}
\hline M & HAM & ADM Pade & Exact & Present \\
\hline 1 & 1.40992 & 1.41421 & 1.41421 & 1.41421 \\
5 & 2.44892 & 2.44948 & 2.44948 & 2.44948 \\
10 & 3.31662 & 3.31662 & 3.31662 & 3.31662 \\
\hline
\end{tabular}

Table 2. Comparison of the skin friction coefficient $-f^{\prime \prime}(0)$ obtained by Keller Box method with HAM, and ADM-Pade solution for some values of $M$, when $\beta=1.5$.

\begin{tabular}{cccc}
\hline M & HAM & ADM Pade & Present \\
\hline 1 & 1.52154 & 1.5252 & 1.5253 \\
5 & 2.51360 & 2.5161 & 2.5162 \\
10 & 3.36672 & 3.3663 & 3.3663 \\
\hline
\end{tabular}

those of Hayat [24] and Abdoul [25]. The comparison revealed good agreement.

Therefore, from the values in Table 1 and Table 2; the code developed for this method can be used with great confidence to study the problem over headed.

Figure 1 and Figure 2 shows the comparison of the solution for the velocity profile obtained by using the Keller box method and the Homotopy analysis method with the exact solution. It is clear from Figure 1 that the graph of the velocity profile $f(\eta)$ coincides exactly with the graph of the exact solution, while it is not in the HAM as shown in Figure 2. This shows that the Keller box method is better than the Homotopy analysis method for this problem. Similarly Figure 3 and Figure 4 show the comparison of the graphs of velocity field $f^{\prime}(\eta)$ obtained by Keller box method and HAM with the exact solution, respectively. It can be observed that the graph of the velocity $f^{\prime}(\eta)$ obtained by Keller box method coincides more to the graph of the exact solution (see Figure 3) than HAM (see Figure 4).

Figure 5 shows the variations of the velocity profile $f(\eta)$ and the velocity field $f^{\prime}(\eta)$ with the similarity variable $\eta$. It illustrates that the velocity profile increases with $\eta$, while the velocity field decreases with an increase in $\eta$. This indicates that the velocity field is maximal at the surface of the plate and reduces as it gets further from the plate and finally becomes zero at a point far away from the plate satisfying the boundary conditions.

Figures 6-8 illustrate effects of the magnetic parameter $M$ and non linearity parameter $\beta$ on the velocity profile $f(\eta)$. It is clear that, as the magnetic parameter $M$ and non-linearity parameter $\beta$ increases the velocity profile $f(\eta)$ decreases. In addition to this the magnetic parameter $M$ has pronounced effect on the decrease of the velocity profile $f(\eta)$ than the non-linearity parameter $\beta$.

Figure 9 and Figure 10 depict the effects of the magnetic parameter $M$ and the non linearity parameter $\beta$ on the velocity field $f^{\prime}(\eta)$, respectively. Figure 9, illustrates that the velocity field $f^{\prime}(\eta)$ decreases with an increase in the magnetic parameter $M$. This is due to the fact that the skin friction at the surface of the plate increases with an increase in $M$ (see the values in Table 1), which in turn reduces the velocity of the fluid. This is because of the induced Lorenz force opposite to the flow.

Figure 10 depicts effect of the nonlinearity parameter $\beta$ on the velocity field $f^{\prime}(\eta)$. It shows that, an increase in the nonlinearity parameter $\beta$ tends to decrease the velocity field $f^{\prime}(\eta)$. Moreover, the effect of the magnetic parameter on the velocity field (see Figure 9) is more pronounced than the effect of the nonlinearity parameter (see Figure 10). The fluid velocity $f^{\prime}(\eta)$ is maximum at the plate surface and decreases exponentially to zero value far away from the plate satisfying the boundary conditions. From these figures, it is noteworthy that the velocity boundary layer thickness decreases with an increase in the values of $M$ and $\beta$.

\section{Conclusions}

In this study, the steady MHD flow of an incompressible viscous fluid over a non-linearly stretching sheet is in- 


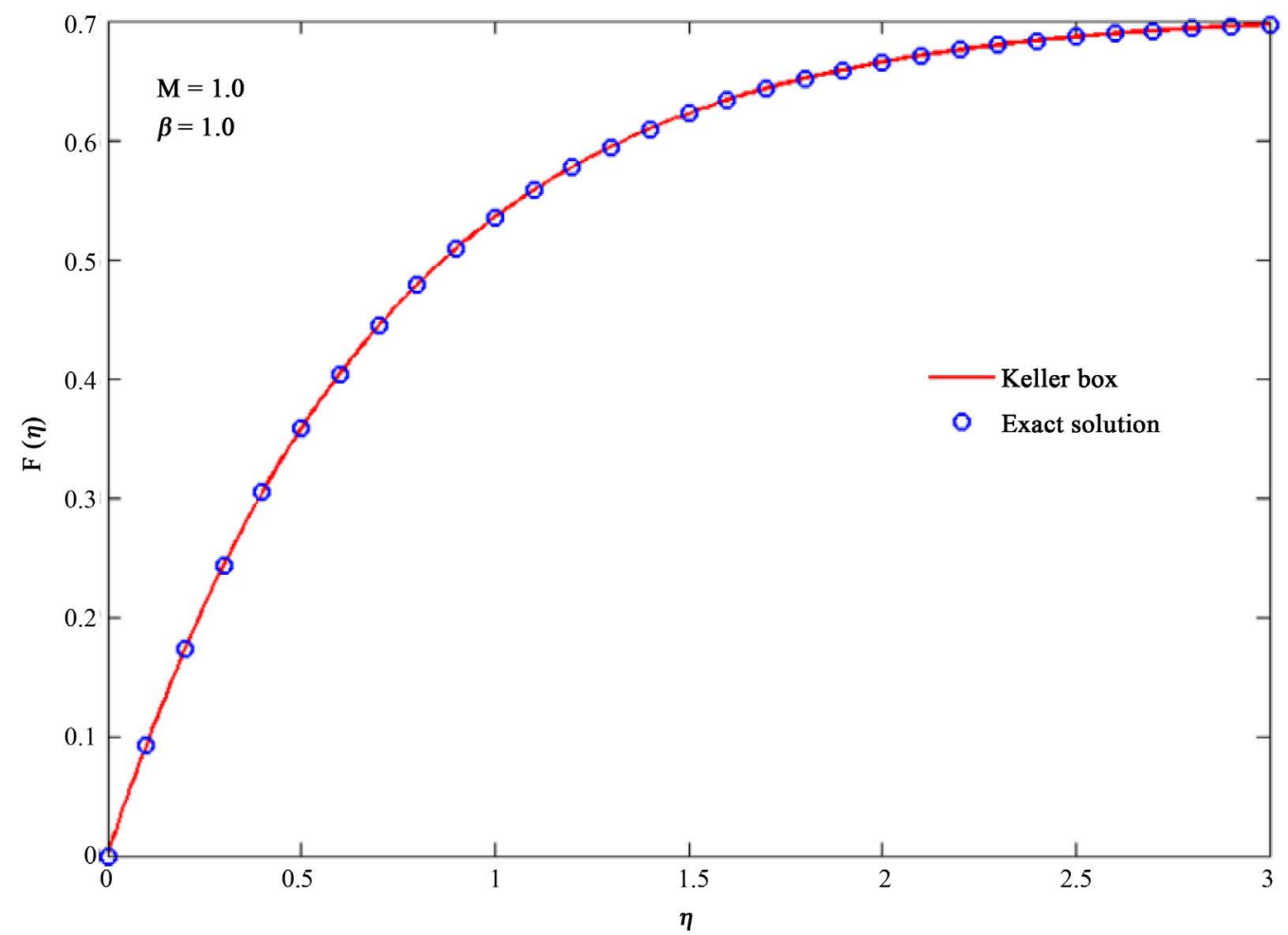

Figure 1. Comparison of velocity profile $f(\eta)$ obtained by the Keller box (Red line) with the Exact solution (circle) for $M=1$ and $\beta=1$.

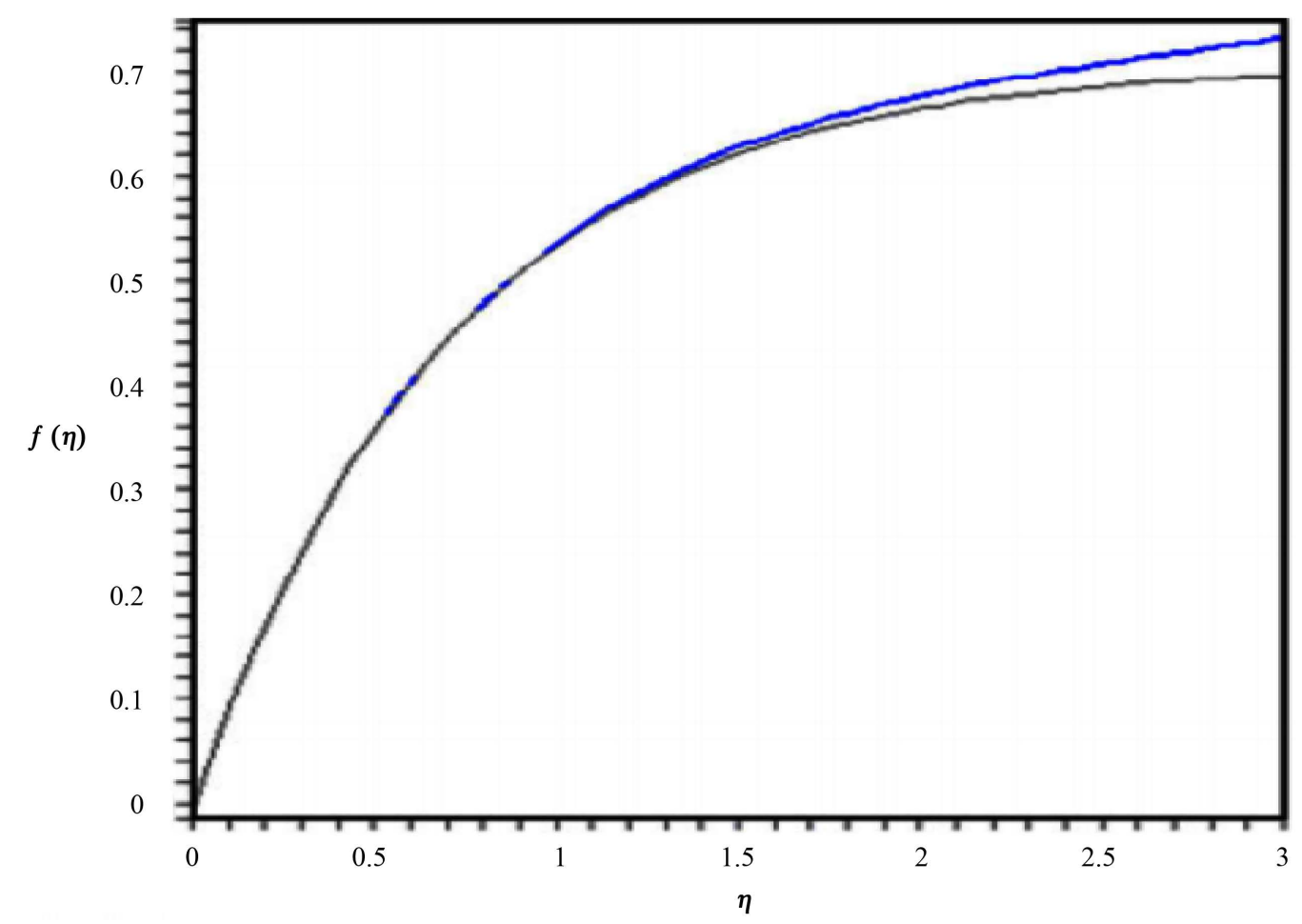

Figure 2. Comparison of velocity profile $f(\eta)$ obtained by the Homotopy Analysis Method (Blue line) with the Exact solution (Black line) for $M=1$ and $\beta=1$ and $h=-0.5$ as in [25]. 


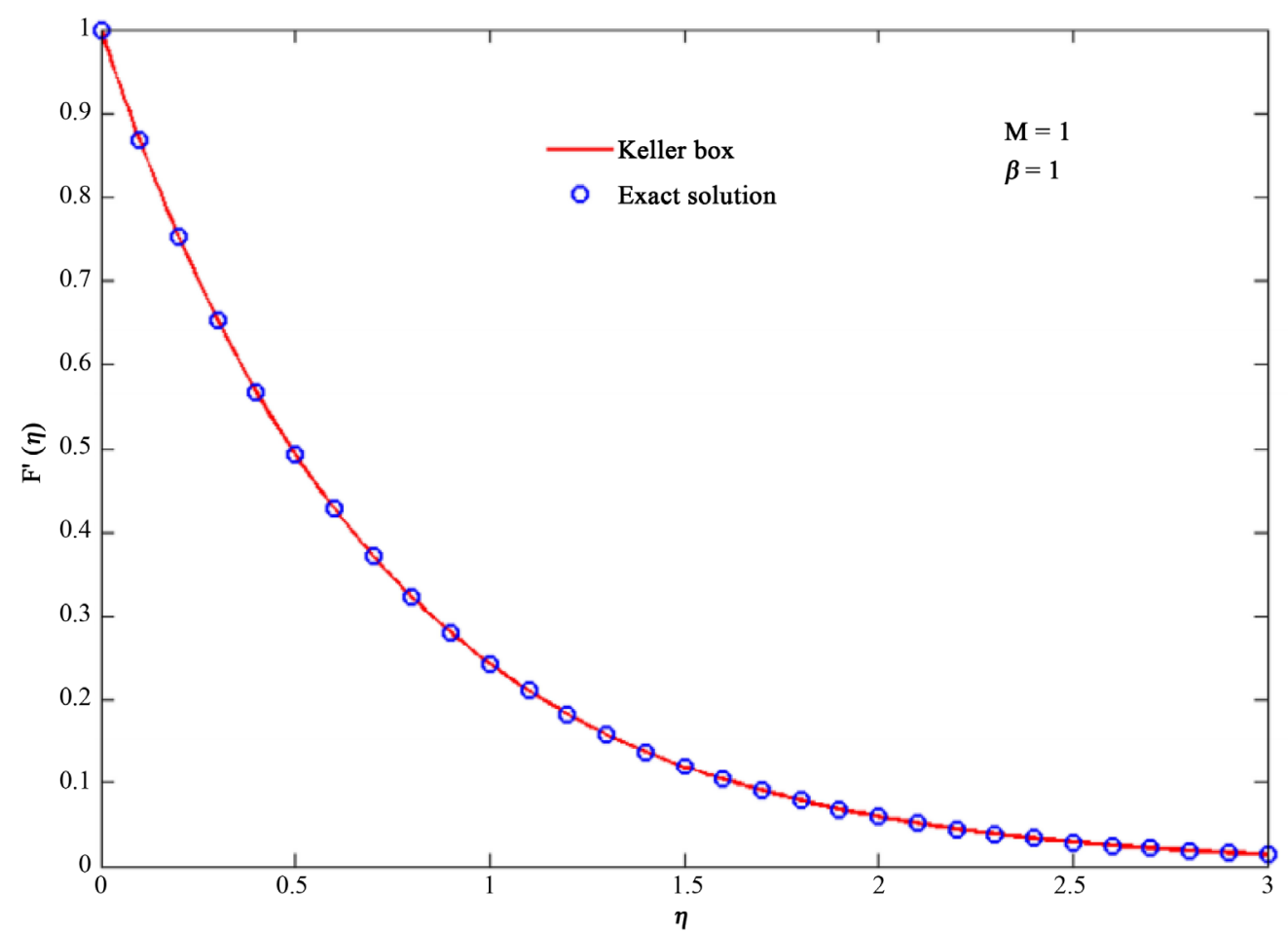

Figure 3. Comparison of velocity field $f^{\prime}(\eta)$ obtained by the Keller box (Red line) with the Exact solution (circle) for $M=1$ and $\beta=1$.

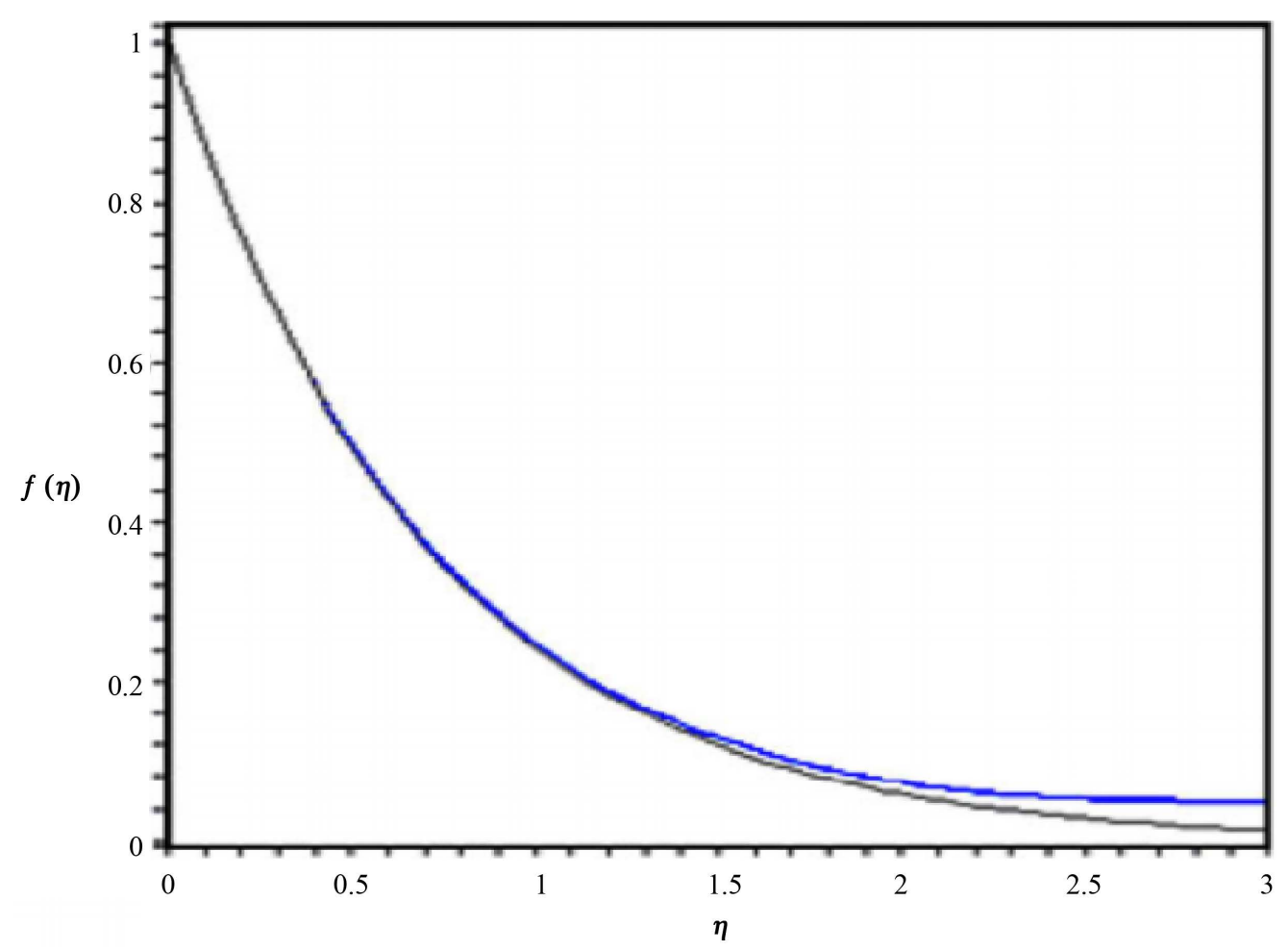

Figure 4. Comparison of velocity field $f^{\prime}(\eta)$ obtained by the Homotopy Analysis Method (Blue line) with the Exact solution (Black line) for $M=1$ and $\beta=1$ and $h=-0.5$ as in [25]. 


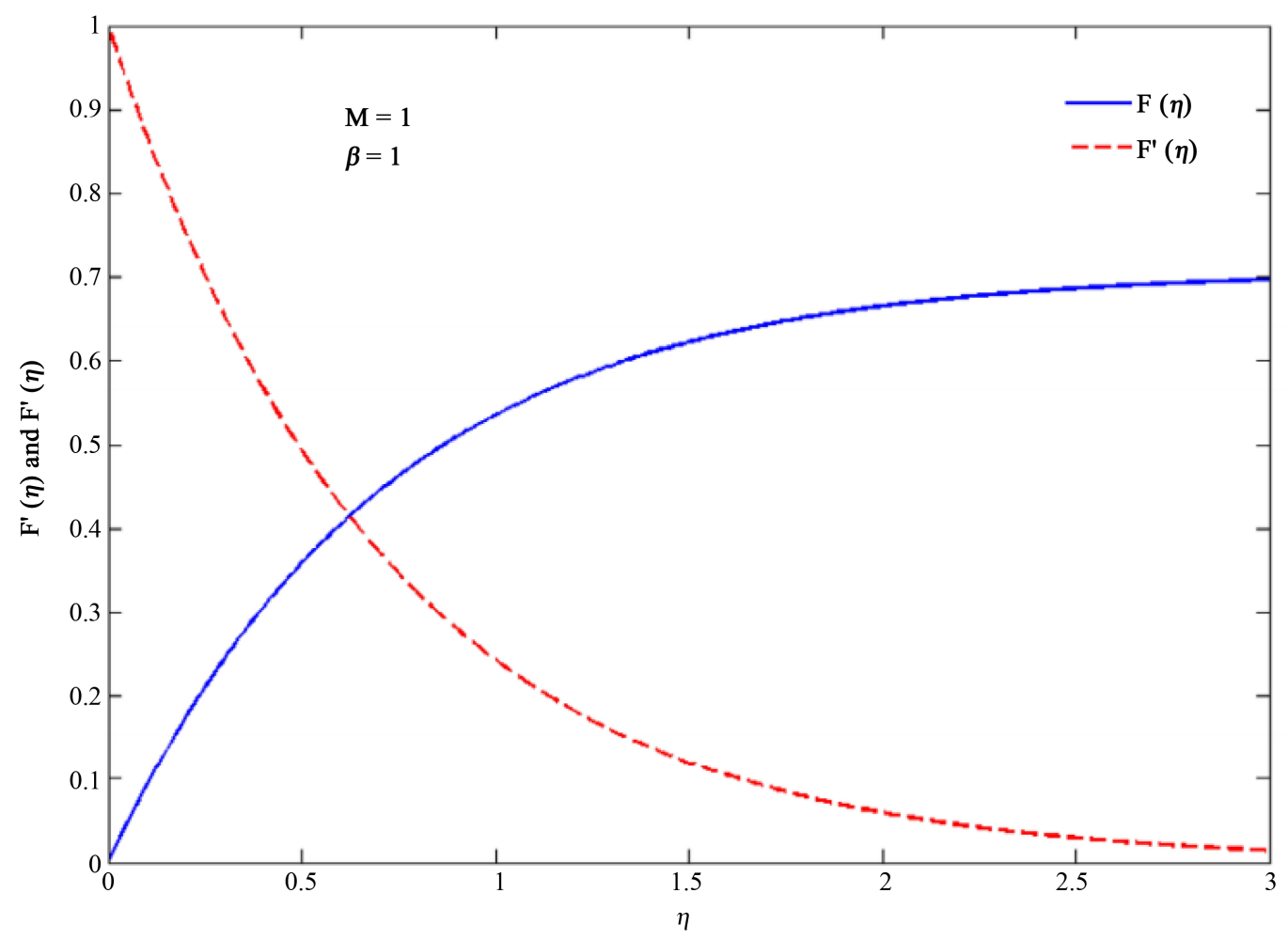

Figure 5. Variation of velocity profile $f(\eta)$ (Blue line) and velocity field $f^{\prime}(\eta)$ (Redline) for $M=1$ and $\beta=1$.

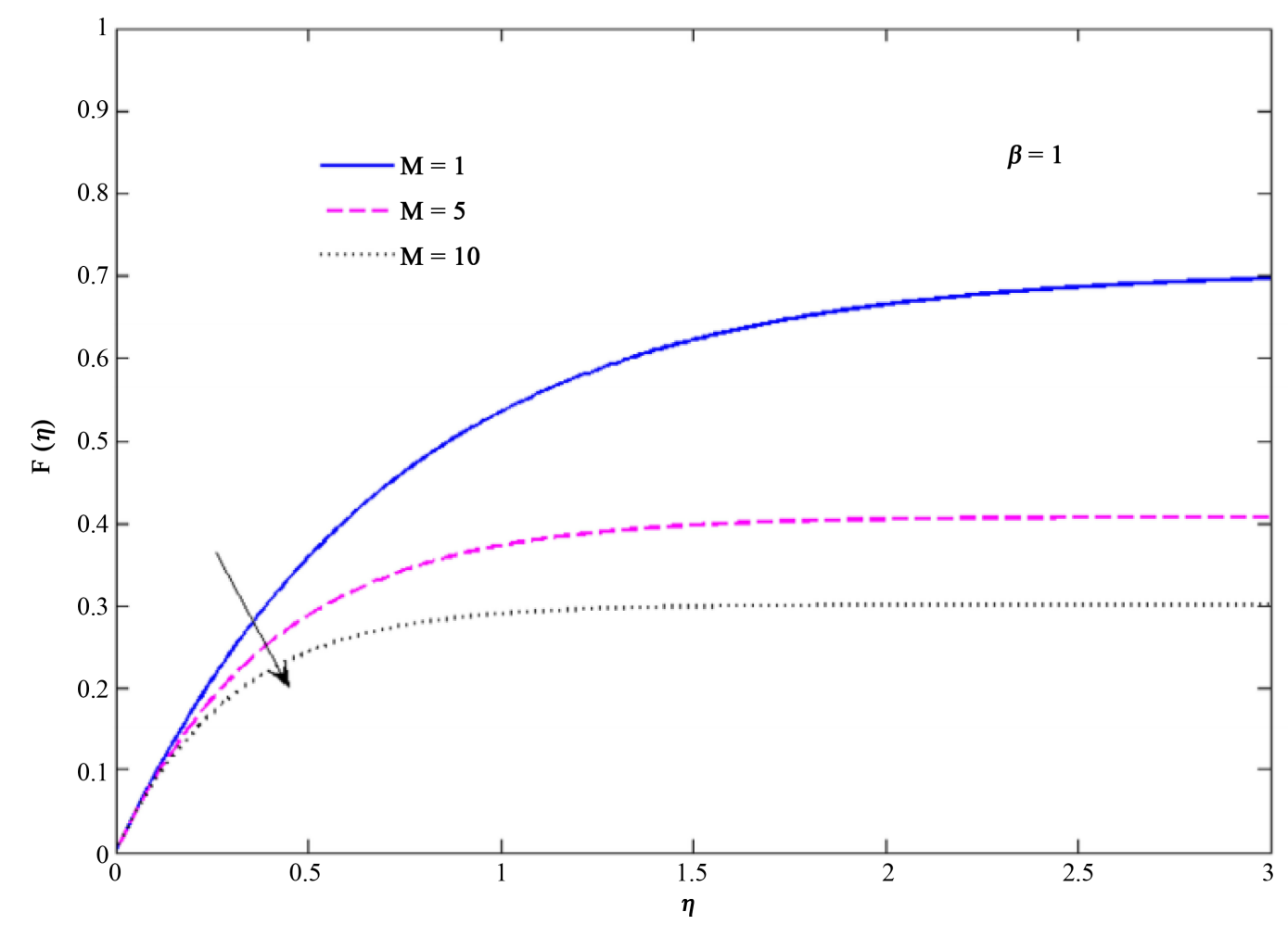

Figure 6. Variation of velocity profile $f(\eta)$ for different values of $M$, when $\beta=1$. 


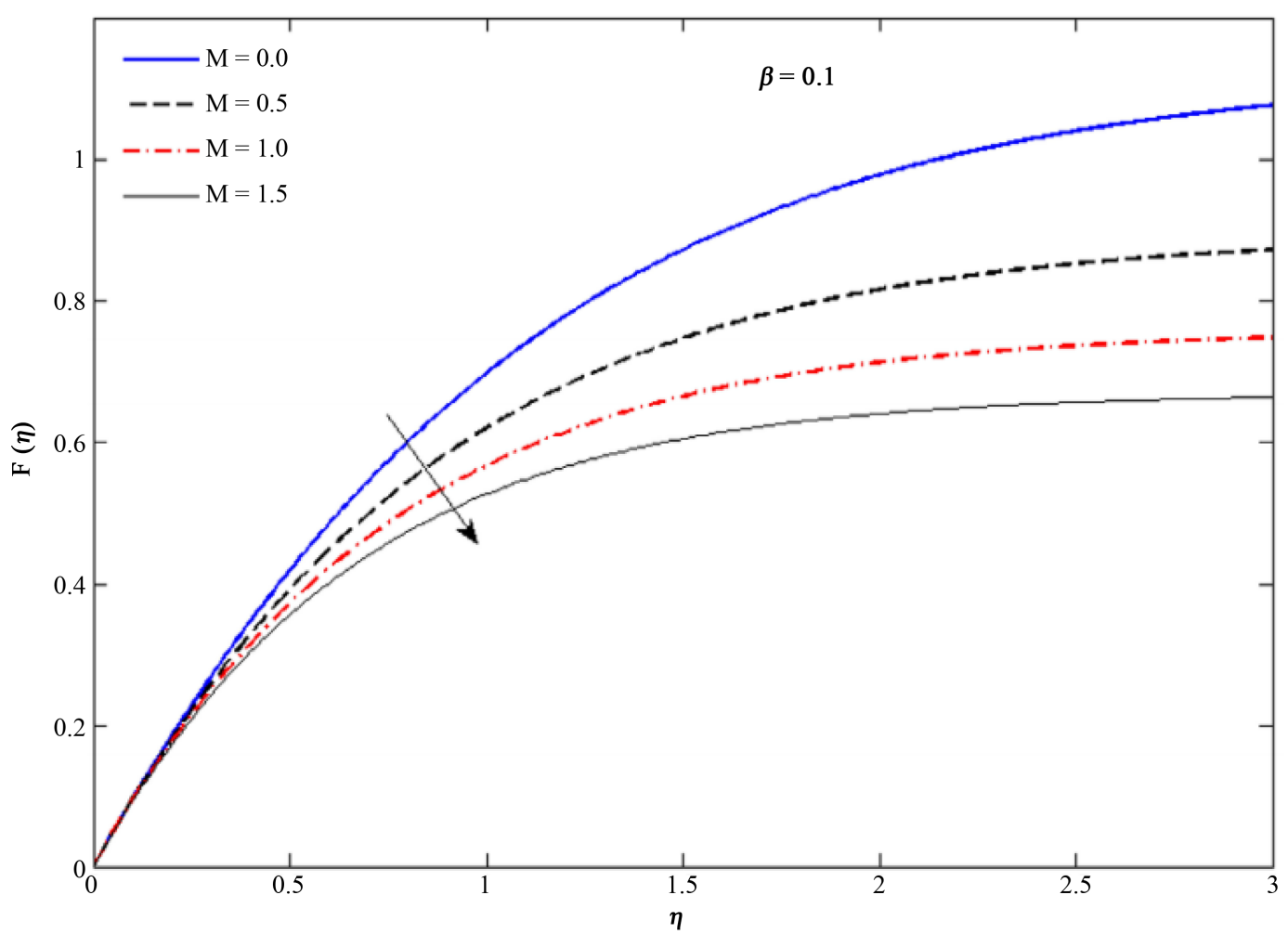

Figure 7. Variation of velocity profile $f(\eta)$ for different values of $M$, when $\beta=0.1$.

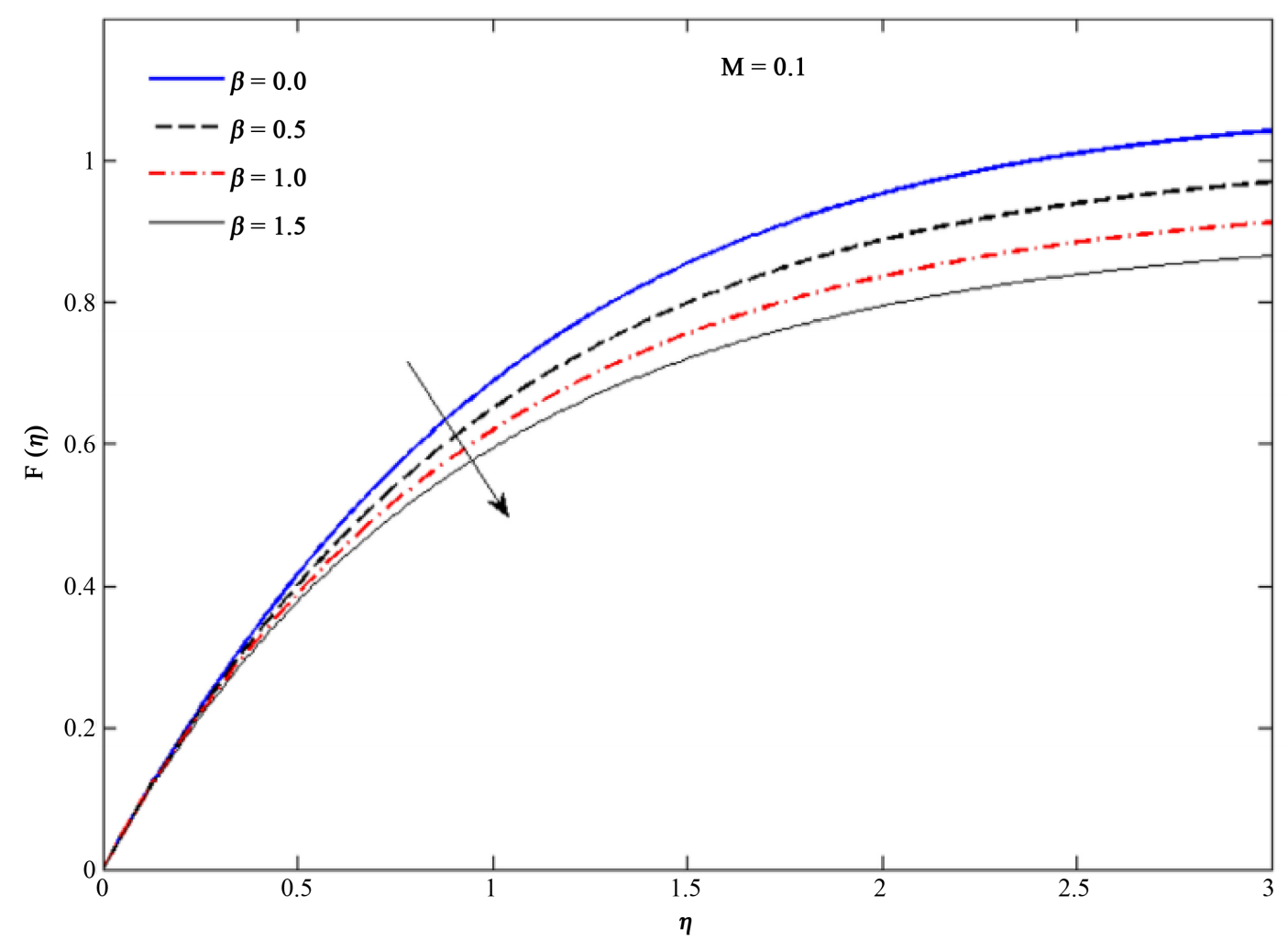

Figure 8. Variation of velocity profile $f(\eta)$ for different values of $\beta$, when $M=0.1$. 


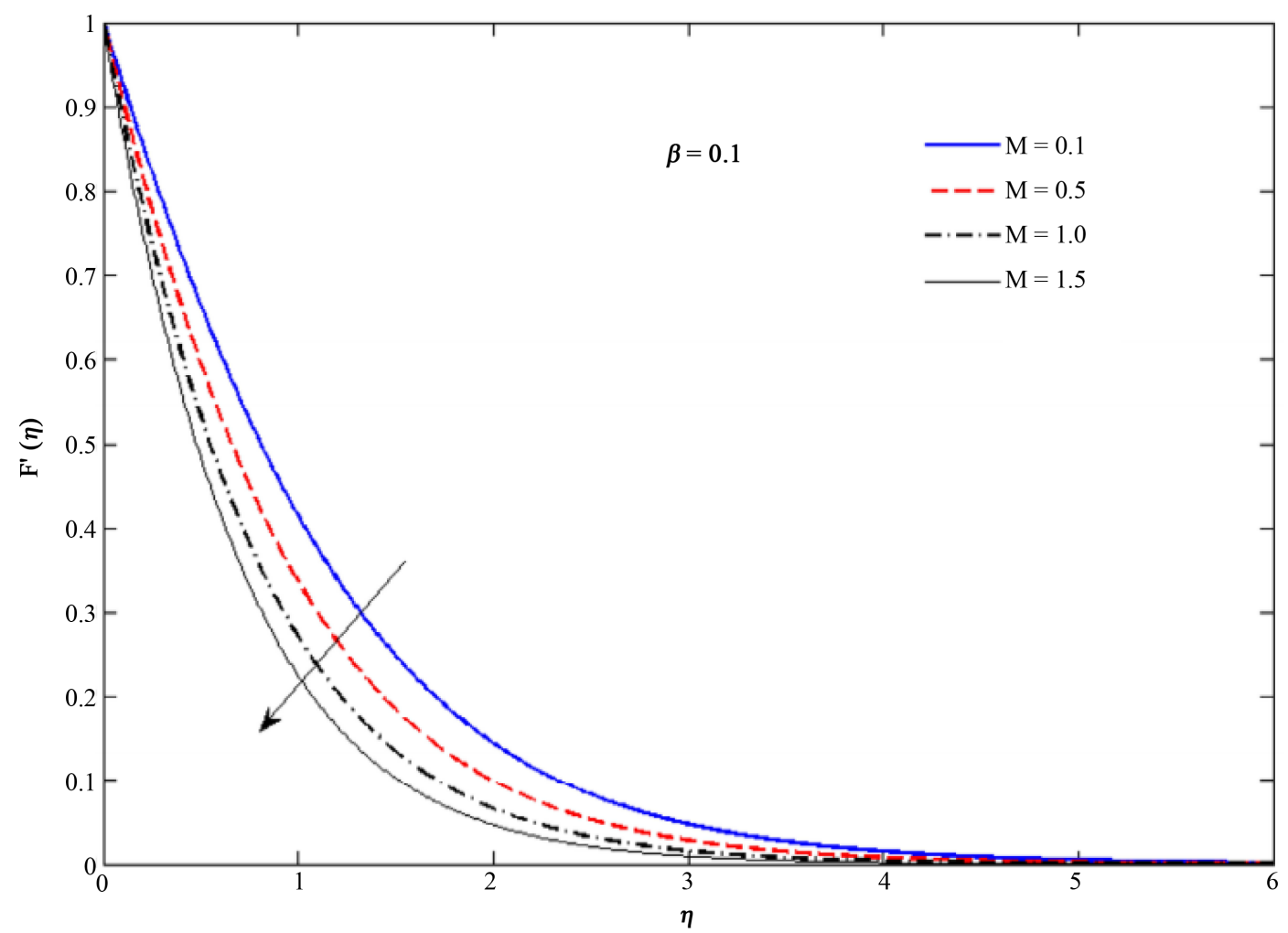

Figure 9. Variation of velocity field $f^{\prime}(\eta)$ for different values of $M$, when $\beta=0.1$.

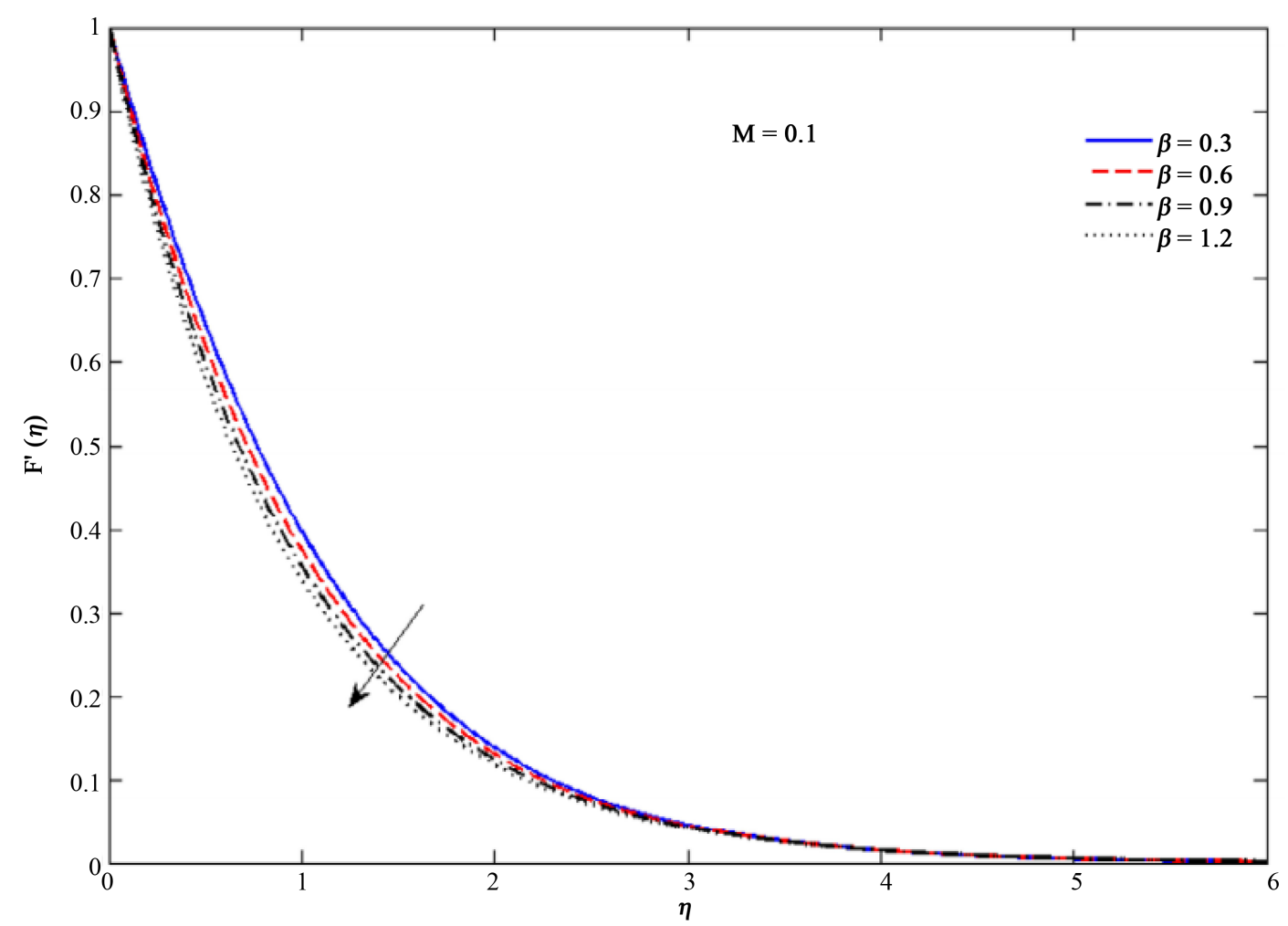

Figure 10. Variation of velocity profile $f^{\prime}(\eta)$ for different values of $\beta$, when $M=0.1$. 
vestigated. A numerical solution using Keller box method is obtained. Besides, for the especial case of $\beta=1$ where the exact solution is available (Equations (9) and (10)). A proper comparison is made between the exact solution, HAM [22], and the present method, Keller box (Figures 1-4 and Table 1 (for - $f^{\prime \prime}(0)$ )).

$$
\begin{aligned}
& f(\eta)=\frac{1-\mathrm{e}^{-\sqrt{1+M \eta}}}{\sqrt{1+M}} \\
& f^{\prime \prime}(0)=-\sqrt{1+M}
\end{aligned}
$$

A comparison of the present study with the existing exact solution and analytic solutions is made for $M=1$ and $\beta=1$. An excellent agreement is achieved with the exact solution. In addition to this the solution obtained using Keller box method is in a better agreement with the exact solution than homotopy analysis method. Moreover, an increase in the non dimensional magnetic parameter $M$ and non linearity parameter $\beta$ causes a decrease in the velocity profile $f(\eta)$, velocity distribution $f^{\prime}(\eta)$ and the velocity boundary layer thickness.

\section{References}

[1] Sakiadis, B.C. (1961) Boundary Layer Behavior on Continuous Solid Surfaces. AIChE Journal, 7, 26-28, http://dx.doi.org/10.1002/aic.690070108

[2] Crane, L.J. (1970) Flow past a Stretching Plate. Zeitschrift für angewandte Mathematik und Physik ZAMP, 21, 645-647. http://dx.doi.org/10.1007/BF01587695

[3] Brady, J.F. and Acrivos, A. (1981) Steady Flow in a Channel or Tube with Accelerating Surface Velocity. An exact solution to the Navier-Stokes Equation with Reverse Flow. Journal of Fluid Mechanics, 112, 127-150. http://dx.doi.org/10.1017/S0022112081000323

[4] Wang, C.Y. (1984) Fluid Flow Due to Stretching Cylinder. Physics of Fluids, 31, 466-468. http://dx.doi.org/10.1063/1.866827

[5] Nadeem, S. (2009) Anwar Hussain: MHD Flow of a Viscous Fluid on a Nonlinear Porous Shrinking Sheet with Homotopy Analysis Method. Applied Mathematics and Mechanics (Engl. Ed.), 30, 1569-1578.

[6] Sajid, M. (2009) Homotopy Analysis of Stretching Flows with Partial Slip. International Journal of Nonlinear Science, 8, 284-290.

[7] Nadeem, S. and Lee, C. (2012) Boundary Layer Flow of Nanofluid over an Exponentially Stretching Surface. Nanoscale Research Letters, 7, 94. http://dx.doi.org/10.1186/1556-276X-7-94

[8] Sharidan, S., Mahmood, T. and Pop, I. (2006) Similarity Solutions for the Unsteady Boundary Layer Flow and Heat Transfer Due to a Stretching Sheet. International Journal of Applied Mechanics and Engineering, 11, 647-654.

[9] Cortell, R. (2007) Viscous Flow and Heat Transfer over a Nonlinearly Stretching Sheet. Applied Mathematics and Computation (Elsevier), 184, 864-873. http://dx.doi.org/10.1016/j.amc.2006.06.077

[10] Alinejad, J. and Samarbakhsh, S. (2012) Viscous Flow over Nonlinearly Stretching Sheet with Effects of Viscous Dissipation. Journal of Applied Mathematics (Hindawi Publishing Corporation), 2012, Article ID: 587834.

[11] Devi, C.D.S., Takhar, H.S. and Nath, G. (1991) Unsteady Mixed Convection Flow in Stagnation Region Adjacent to a Vertical Surface. Heat and Mass Transfer, 26, 71-79.

[12] Andersson, H.I., Aarseth, J.B. and Dandapat, B.S. (2000) Heat Transfer in a Liquid Film on an Unsteady Stretching Surface. International Journal of Heat and Mass Transfer, 43, 69-74. http://dx.doi.org/10.1016/S0017-9310(99)00123-4

[13] Nazar, R., Amin, N. and Pop, I. (2004) Unsteady Boundary Layer Flow Due to Stretching Surface in a Rotating Fluid. Mechanics Research Communications, 31, 121-128. http://dx.doi.org/10.1016/j.mechrescom.2003.09.004

[14] Elbashbeshy, E.M.A. and Bazid, M.A.A. (2004) Heat Transfer over an Unsteady Stretching Surface. Heat and Mass Transfer, 41, 1-4. http://dx.doi.org/10.1007/s00231-004-0520-x

[15] Ishak, A., Nazar, R. and Pop, I. (2009) Heat Transfer over an Unsteady Stretching Permeable Surface with Prescribed Wall Temperature. Nonlinear Analysis: Real World Applications, 10, 2909-2913. http://dx.doi.org/10.1016/j.nonrwa.2008.09.010

[16] Wubshet, I., Shankar, B. and Nandeppanavar, M.M. (2013) MHD Stagnation Point Flow and Heat Transfer Due to Nanofluid towards Astretching Sheet. International Journal of Heat and Mass Transfer, 56, 1-9.

[17] Turkyilmazoglu, M. and Pop, I. (2013) Heat and Mass Transfer of Unsteady Natural Convection Flow of Some Nanofluids Past Avertical Infinite Flat Plate with Radiation Effect. International Journal of Heat and Mass Transfer, 59, 
167-171. http://dx.doi.org/10.1016/j.ijheatmasstransfer.2012.12.009

[18] Xu, H., Pop, I. and You, X.C. (2013) Flow and Heat Transfer in a Nano-Liquid Film over an Unsteady Stretching Surface. International Journal of Heat and Mass Transfer, 60, 646-652. http://dx.doi.org/10.1016/j.ijheatmasstransfer.2013.01.046

[19] Lyapunov, A.M. (1992) General Problem on Stability of Motion (English Translation). Taylor and Francis, London.

[20] Karmishin, A.V., Zhukov, A.I. and Kolosov, V.G. (1990) Methods of Dynamics Calculation and Testing for ThinWalled Structures. Mashinostroyenie, Moscow.

[21] Adomian, G. (1994) Solving Frontier Problems of Physics: The Decomposition Method. Kluwer Academic Publishers, Boston and London.

[22] Liao, S.J. (1992) The Proposed Homotopy Analysis Technique for the Solution of Nonlinear Problems. PhD Thesis, Shanghai Jiao Tong University, Shanghai.

[23] Keller, H.B. (1971) A New Difference Scheme for Parabolic Problems. In: Hubbard, B., Ed., Numerical Solutions of Partial Differential Equations, II, Academic Press, New York, 327-350.

[24] Hayat, T., Hussain, Q. and Javed, T. (2007) The Modified Decomposition Method and Pade Approximation for the MHD Flow over a Non-Linear Stretching Sheet. Nonlinear Analysis: Real World Applications, 10, 966-973.

[25] Ghotbi, A.R. (2009) Homotopy Analysis Method for Solving the MHD Flow over a Non-Linear Stretching Sheet. Communications in Nonlinear Science and Numerical Simulation, 14, 2653-2663.

[26] Cebeci, T. and Pradshaw, P. (1988) Physical and Computational Aspects of Convective Heat Transfer. Springer, New York. http://dx.doi.org/10.1007/978-1-4612-3918-5 